Article available at http://Www.parasite-journal.org or nttp://dx.dol.org/10.1051/parasite/2004173249

\title{
DeuX puces nouvelles du ChILI et d'ARGentine (INSECTA-SIPHONAPTERA : StePHANOCIRCIDAE ET RHOPALOPSYLLIDAE) ET ÉRECTION D'UN SOUS-GENRE CHEZ PLOCOPSYLLA JORDAN 1931
}

\author{
BEAUCOURNU J.C.******, GALLARDO M.H.** \& MÉNIER K.****,******
}

Summary: TWO NEW SPECIES OF FLEAS FROM CHILE AND ARGENTINA and RaIsing of a New sub-genus of PlocopsyLLA Jordan, 1931

A new subgenus of Plocopsylla is raised, Schrammapsylla Beaucournu and Ménier from the entire group B of Schramm \& Lewis, 1988 and two new fleas from Chile and Argentina are described: Plocopsylla (S.) muruai n. sp. Beaucournu and Gallardo and Ectinorus (E.) mimacydis n. sp. Beaucournu and Gallardo. These two taxa are only known by the male sex.

KEY WORDS : Schrammapsylla n. subg. of Plocopsylla, Plocopsylla (S.) muruai n. sp, Ectinorus (E.) mimacydis n. sp.

\section{INTRODUCTION}

I a faune des puces de la région chilio-andine ne cesse de s'accroître. Nous décrivons ici deux espènouvelles, une Plocopsylla Jordan, 1931 (et en profitons pour ériger les groupes A et B de Schramm \& Lewis (1988) en sous-genres) et un Ectinorus Jordan, 1942.

\section{DESCRIPTION}

- Famille Stephanocircidae

Genre Plocopsylla Jordan, 1931

Ce genre chilio-andin, essentiellement parasite de rongeurs, est riche de 28 espèces décrites. Nous en ajoutons une nouvelle et en profitons pour discuter du statut de ces taxa. Se basant surtout, mais non uniquement, sur la structure du télomère ("movable process", auct.), Schramm \& Lewis (op. cit.) scindent ce

\footnotetext{
* Laboratoire de parasitologie, Faculté de médecine, Avenue du Professeur Léon Bernard, F-35043 Rennes cedex, France.

** Instituto de ecologia y evolucion, Universidad Austral de Chile, Casilla 567, Valdivia, Chile.

*** Institut de parasitologie de l'Ouest, Avenue du Professeur Léon Bernard, F-35043 Rennes cedex, France.

***** School of veterinary medicine, Faculty of medical sciences, Eric William Medical Sciences Complex, Champs Fleurs, St-Augustine, Trinidad and Tobago, The West Indies.

Correspondance : Jean-Claude Beaucournu.

E-mail : jbeaucou@univ-rennes1.fr
}

\begin{abstract}
Résumé :
Les auteurs érigent le groupe B (Schramm \& Lewis, 1988) du genre Plocopsylla Jordan, 1931 en sous-genre nouveau, Schrammapsylla Beaucournu et Ménier. Par ailleurs, ils décrivent Plocopsylla (S.) muruai n. sp. Beaucournu et Gallardo et Ectinorus (E.) mimacydis Beaucournu et Gallardo. Les deux taxons ne sont connus que par le sexe mâle.
\end{abstract}

MOTS CLÉS : Schrammapsylla n. subgen. de Plocopsylla, Plocopsylla (S.) murua n. sp., Ectinorus (E.) mimacydis n. sp. genre en deux groupes A et $\mathrm{B}$. Le groupe A se subdivise en quatre sous-groupes (A1-A4) et le groupe B, en deux (B1-B2). Le problème des groupes a fait couler déjà beaucoup d'encre. Smit (1963), comme certains auteurs, estimait que la création de sous-genres figeait la taxonomie dans son aspect évolutif et préférait, dans sa magnifique Monographie du genre Ctenophthalmus, parler de groupes et sous-groupes. En 1966, Hopkins \& Rothschild, dans le tome IV du Catalogue of the Rothschild Collection of Fleas, tome consacré dans sa majeure partie à ce genre, écrivaient : "We entirely agree with Smit... but... the gratus subgroup of the assimilis group are not preferable to the gratus group of Euctenophthalmus. We have, therefore, used subgeneric names for the most of the group...". Smit, lui-même, se rallie à cette position pour les nouveaux Ctenophthalmus qu'il décrit par la suite. Il fera de même dans sa Monographie (volume VII du Catalogue, 1987) sur les Malacopsylloidea. Schramm \& Lewis (1988) ont éprouvé le même scrupule, et c'est à leur honneur, à propos du genre Plocopsylla. Nous pensons cependant que, pour le lecteur, lire Plocopsylla (Plocopsylla) achilles Jordan, 1931 situe mieux la position de la puce, que $P$. achilles Jordan, 1931, groupe A1, sensu Schramm \& Lewis (1988). C'est pourquoi, après avoir communiqué notre position à ces deux auteurs, nous élevons les groupes A et B (les sousgroupes deviennent, ipso facto, des groupes) en :

- sous-genre Plocopsylla, espèce-type P. achilles (cf. Jordan, 1931) = "groupe A" ;

- sous-genre Schrammapsylla Beaucournu et Ménier, n. subgen., espèce type P. wolffsohni (Rothschild, 1909) = "groupe B". 
Nous sommes heureux de pouvoir rendre cet hommage à Beth Schramm pour sa thèse remarquable soutenue en 1987, et effectuée sous l'égide de notre ami le Professeur R.E. Lewis (Université de l'Iowa).

Deux espèces nouvelles s'intègrent dans le sous-genre Plocopsylla (ex groupe A) : P. silewi Beaucournu et Kelt, 1990 et $P$. wilesi Beaucournu et Kelt, 1990. De même, par rapport au travail de Schramm \& Lewis (1988), sont à ajouter à leur liste (p. 7), pour le sous-genre Schrammapsylla, trois espèces, toutes appartenant au groupe 1 : - P. crypta Beaucournu et Gallardo, 1989,

- P. consobrina Beaucournu et Alcover, 1990,

- Plocopsylla n. sp., présent travail.

Nous renvoyons au travail précité de Schramm \& Lewis pour une définition complète de ce sous-genre (en tant que groupe $\mathrm{B}$ ). Les deux premières espèces citées ci-dessus sont référencées in Beaucournu \& Gallardo (1991).

\section{PLOCOPSYLLA (SCHRAMMAPSyLLA) MURUAI N. SP. Beaucournu et Gallardo}

Matériel de description : holotype mâle (et unique exemplaire) sur Abrothrix (= Akodon) olivaceus (Waterhouse, 1837) (Muridae, Sigmodontinae), Parc national de Cucao (lat. : $42^{\circ} 38^{\prime} \mathrm{S}-$ long. : $74^{\circ} 07^{\prime} \mathrm{W}$ ), 9 août 1995 , Île et Province de Chiloë, Chili, Roberto Murua et Freddy Mondaca rec. (n 52 RM).

Dépot du type : dans les collections du premier auteur, Faculté de médecine, Rennes, ultérieurement déposées au Laboratoire d'entomologie du Muséum national d'histoire naturelle de Paris.

Derivatio nominis : nous sommes heureux de dédier ce nouveau taxon à R. Murua, pour sa précieuse collaboration.

Description : basimère unique dans le sous-genre par sa forme et la disposition de sa frange de longues soies; télomère allongé, avec l'extrémité basale arrondie en pommeau de canne, l'extrémité apicale courbée ventralement, dotée de grosses soies spiniformes foliacées et échancrées, l'inférieure très sclérotisée.

Capsule céphalique (figure 1) : partie précténidiale du casque ("helmet") aussi large que les $2 / 3$ de la plus longue épine (la $5^{\text {jeme})}$ de cette cténidie. Onze épines sur ce peigne, ayant toutes une nette encoche basale, sauf la supérieure et les deux inférieures. Cténidie génale de cinq épines, toutes longues; la supérieure est légèrement plus courte, plus acuminée et plus étroite que la suivante, dont elle est séparée par un espace inférieur à la moitié de sa propre largeur basale. Apex du lobe génal élargi et arrondi. La gena, dans son ensemble, est nettement plus longue que haute comme, par exemple, chez $P$. (S.) crypta. Soies du pédicelle antennaire atteignant la moitié de la massue. Partie antéro-occipitale de la capsule céphalique por- tant, dans sa partie juxta-post-cténidiale, plusieurs soies petites et deux rangs de quatre et cinq soies, le dernier rang composé de soies plus longues; la partie postéro-occipitale porte trois rangs de cinq, trois et sept soies (pour le rang distal). Palpe labial, composé de cinq articles, atteignant l'apex de la coxa I.

Thorax : prothorax, avec deux rangs de soies et une soie antérieure d'une rangée vestigiale; cténidie de 18 épines. Mésothorax : deux rangées de soies et une antérieure vestigiale; une pseudo-seta dorsale. Métathorax : deux rangées de soies. Pseudo-peignes particulièrement denses sur les tibias I et II; la plus grande soie du tibia III atteint l'apex du premier segment tarsal. Abdomen (segments non génitaux) : chétotaxie des tergites I à V comparables, quelques soies courtes et une rangée de soies longues : huit dont une au dessous du stigmate. Spinules marginales présentes sur les tergites I-V : 5, 2, 3, 2, 1. Stigmates arrondis. Soie antésensiliale sur un piédestal. Apophyse antérograde du tergite VIII longue. Sternites: II , avec une soie, III à VI avec systématiquement deux soies d'un côté et trois de l'autre (ce qui est, bien sûr, un caractère propre à ce spécimen); sternite VII sans sétation décelable.

Abdomen (segments génitaux) : segment VIII : tergite membraneux, hyalin, difficile à discerner, apparaissant plus ou moins arrondi; sternite quadrangulaire, plus étroit sur son bord antérieur que postérieur, celui-ci étant légèrement convexe dans ses $2 / 3$ supérieurs, puis concave dans sa partie basale. Segment IX (figure 2) : basimère unique dans ce sous-genre par sa forme et l'implantation de la rangée de grandes soies sur la marge dorso-postérieure. Chez les autres espèces du sous-genre Schrammapsylla, c'est sur un bord distal largement arrondi, y compris postérieurement, que sont situées ces soies. La zone sétifère est à peine convexe, le bord ventral est, dès l'angulation, doucement convexe, puis légèrement concave. Le processus digitiforme est en partie masqué par la $2^{\text {ème }}$ grande soie : il est subquadrangulaire, large, doucement arrondi à son apex. Le processus interne à la forme classique en "boomerang"; ses deux extrémités sont doucement élargies et le bord inféro-distal nettement concave. Télomère de structure classique dans le sous-genre, les différences interspécifiques sont essentiellement au niveau des grosses soies spatulées et/ou spiniformes : soie supérieure (à l'apex du processus) incolore, double, car scindée en deux parties évoquant les ailes ouvertes d'un papillon; soie inférieure, fortement sclérotisée, formée elle aussi de deux parties, la plus dorsale large, longue, tortueuse, alors que la ventrale est fine et se termine en spirale courte. Sternite de forme générale classique du sous-genre : apex arrondi; trois soies à sa partie supéro-distale (comme d'ailleurs chez P. (S.) crypta, ce qui n'est pas mentionné dans la description du type), soie supérieure spatulée et hyaline 


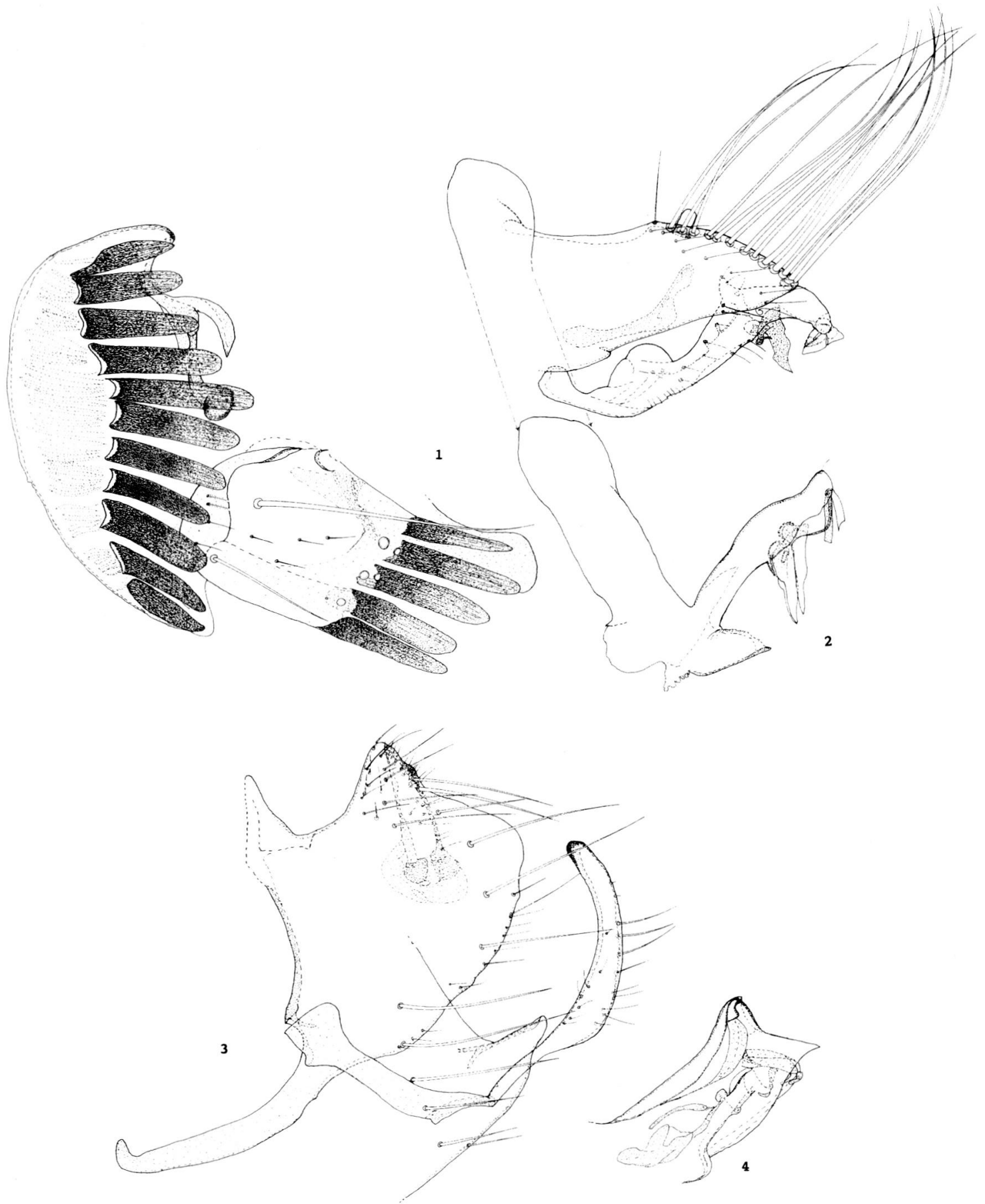

Fig. 1. - Plocopsylla (Schrammapsylla) muruai Beaucournu et Gallardo, capsule céphalique (partie antérieure) et gena de l'holotype. Fig. 2. - P. (S.) muruai, segment IX de l'holotype.

Fig. 3. - Ectinorus (E.) mimacydis Beaucournu et Gallardo : sternite VIII et segment IX de l'holotype.

Fig. 4. - E. (E.) mimacydis, apex de l'aedeagus de l'holotype.

en forme de lame de "navaja" (poignard espagnol), les deux suivantes se recouvrent totalement à la base : la plus interne spatulée, peu colorée, quadrangulaire (mais il est possible qu'une superposition de soies, épines, organes... interfère dans la forme que nous lui attribuons); la plus externe, fortement pigmentée, séti- forme sinueuse et facilement confondue avec le contour postéro-apical du bras distal de ce sternite. Au-dessous, deux grosses soies spatulées, l'inférieure semblant déboublée; près de la base de chacune, légèrement pigmentée, on note une petite formation constituée de points oblongs rangés en forme d'ovale. Nous n'avons 
pu élucider la nature exacte de ces "points". Phallosome : apex du ductus courbé vers l'arrière avec, comme il est fréquent, un très léger évasement de l'orifice.

Femelle inconnue.

Dimension (insecte monté) : mâle holotype $2,10 \mathrm{~mm}$ (sans les soies marginales du basimère).

\section{Discussion}

Espèce typique de Schrammapsylla, immédiatement différenciable des autres taxa de ce sous-genre par la forme du basimère.

- Famille Rhopalopsyllidae

\section{ECTINORUS (ECTINORUS) MIMACYDIS N. SP. Beaucournu et Gallardo}

Matériel de description : mâle holotype (en compagnie de dix Delostichus incisus Beaucournu et Torrès-Mura, 1988 sur Octomys mimax Thomas, 1920, Parc National Ischigualasto (lat. : $31^{\circ} \mathrm{S}-$ long. : $68^{\circ} \mathrm{W}$ ), 9 août 2002 , Prov. San Juan, Argentine, M.H.G.rec. ( ${ }^{\circ} 1683$ ). Cette station est la terra typica de D. incisus, collecté, d'ailleurs, sur le même hôte.

Dépôt du type : ( $c f$. ci-dessus).

Derivatio nominis : de Octomys mimax, seul hôte connu. Description : depuis la Monographie de Smit (1987), cinq taxa nouveaux, dont celui-ci, furent intégrés dans le genre et le sous-genre Ectinorus : E. gallardoi Hastriter, 2001 (Chili), E. moncadai Hastriter, 2001 (Chili), E. pilosus Beaucournu et del Carmen Castro, 2002 (Argentine), E. alejoi Hastriter, 2002 (Pérou) et l'espèce suivante, d'Argentine. Aucune de ces espèces ne fait partie du complexe possédant un "processus basimeris ventralis" individualisé.

Capsule céphalique : oeil bien développé. Soies du pédicelle atteignant seulement la base de la massue antennaire. Apex du lobe génal très doucement incurvé. Soies céphaliques très peu nombreuses : un rang d'une soie et le rang occipital de cinq soies. Palpe labial de six articles au moins (l'insecte mal fixé est de "lecture" difficile).

Thorax : une rangée de soies sur chaque segment avec une pseudo-setae sous le collier du mesonotum, et deux sous le collier du metanotum.

Abdomen (segments non génitaux) : une spinule marginale sur le tergite I; aucune sur les autres. Cinq soies longues sur les tergites II-VI. Soies antésensiliales semblant être sur un piédestal, mais celui-ci est en fait réuni au reste du segment par une membrane hyaline; leur longueur n'est pas connue, les deux soies étant arrachées. Sternites III à VII avec deux à trois soies, espacées. Sternite VIII (figure 3) montrant un lobe ventral sclérotisé, puis une encoche suivie d'une marge convexe; il porte six soies, dont une petite marginale.
Abdomen (segments génitaux) : tergite IX (figure 3) : basimère sans bras basal ("processus basimeris ventralis"); apex du basimère en triangle équilatéral se continuant en pente oblique jusqu'à la courbure postéroventrale. Manubrium étroit, presque aussi long que la largeur du basimère, à apex retroussé. Télomère long, étroit, acuminé au sommet, mais à bords sub-parallèles jusqu'aux $4 / 5^{\text {èmes }}$ de sa longueur. Sternite IX (figure 3) long et grêle, particulièrement à la jonction des parties basale et distale; partie distale à bord parallèles et à chetotaxie réduite.

Phallosome: nous ne représentons que l'apex de l'aedeagus (figure 4).

Femelle inconnue.

Dimensions (insecte monté mais légèrement contracté) : mâle holotype $1,4 \mathrm{~mm}$.

\section{RÉFÉRENCES}

Beaucournu J.C. \& Gallardo M.H. Catalogue provisoire des puces du Chili (Insecta : Siphonaptera) (1 ${ }^{\mathrm{re}}$ partie). Bulletin de la Société Française de Parasitologie, 1991, 9, $237-$ 270 .

Beaucournu J.C. \& Kelt D.A. Contribution à la faune du Chili : puces nouvelles ou peu connues de la partie sud (InsectaSiphonaptera). Revue Suisse de Zoologie, 1990, 97, 647-668.

Hastriter M.W. Fleas (Siphonaptera: Ctenophthalmidae and Rhopalopsyllidae) from Argentina and Chile with two new species from the Rock Rat, Aconaemys fuscus, in Chile. Annals of Carnegie Museum, 2001, 70, 169-178.

Hastriter M.W., Zyzak M.D., Soto R., Fernandez R., SolorzANo N. \& Whiting M.F. Fleas (Siphonaptera) from Ancash department, Peru with the description of a new species, Ectinorus alejoi (Rhopalopsyllidae), and the description of the male of Plocopsylla pallas (Rothschild, 1914) (Stephanocircidae). Annals of Carnegie Museum, 2002, 71, 87-106.

Hopkins G.H.I. \& Rothschild M. An illustrated catalogue of the Rothschild collection of Fleas (Siphonaptera) in the British Museum, Vol. IV, British Museum, London, 1966, 549 p. $+12 \mathrm{pl}$

JORDAN K. Further records and descriptions of fleas from Ecuador. Novitates Zoologicae, 1931, 37, 135-143.

Schramm B.A. \& LewIS R.L. Revision of Plocopsylla (Siphonaptera: Stephanocircidae). Koenigstein, Koelts Scientific Bookes, 1988, 157 p.

Smit F.G.A.M. Species-groups in Ctenophthalmus (Siphonaptera: Hystrichopsyllidae). Bulletin of the British Museum (Natural History) Entomology, 1963, 14, 152 p.+ 58 pl.

Smit F.G.A.M. An illustrated Catalogue of the Rothschild Collection of Fleas, Vol. VII., Malacopsylloidea. Oxford University Press, The British Museum (Natural History), 1987, 380 p. +5 pl.

Reçu le 26 janvier 2004

Accepté le $1^{\text {er }}$ avril 2004 